\title{
Jan Hodel \\ A Historyblogosphere Of Fragments. Überlegungen zum fragmentarischen Charakter von Geschichte, von Blogs und von Geschichte in Blogs
}

Dieser Beitrag, der den fragmentarischen Charakter von Blogs und die historiographischen Konsequenzen daraus behandelt, gestattet sich eine leicht fragmentarische Form der Darstellung und des Aufbaus. Diese andeutungsweise Annäherung an ein fragmentarisches Format, die im Folgenden als für Weblogs typisch vorgestellt wird, soll die Erwartungshaltung der Lesenden herausfordern und - es sei offen deklariert - für die Argumente am Ende des Textes empfänglich machen. Dass dieser versuchsweise Umgang nur eine Andeutung bleibt, und beispielsweise auf eine chronologisch umgekehrte Darstellung (den Schluss zuerst, den Anfang am Ende) verzichtet wird, mögen die geneigten Leserinnen und Leser dem Autor nachsehen.

Der Begriff des Fragments will für eine Beschreibung digitaler Medienwirklichkeit nicht recht passen. Er impliziert Vorstellungen von Unvollständigkeit, von Verlust und Reduktion, die nicht passen wollen zu den Kernkonzepten digitaler Medien - wie der Medienintegration, der verlustfreien Duplikation von Daten, der technischen Unterstützung sozialer Interaktion im Social Web, dem daraus resultierenden produktiven Zusammenwirken zahlreicher Menschen im kollaborativen Prozess des Crowdsourcing, dem Informationsüberfluss und, vor allem, der Vernetzung. Das einzelne Element, sei es Informationseinheit oder menschlicher Akteur, wird im digitalen Zeitalter als Modul bezeichnet, dessen Merkmal seine Verbindung mit anderen Modulen ist, die sich idealiter zu einem Netz anordnen. Ausgreifender noch als bei der Metapher des Netzes, das immerhin leere Zwischenräume kennt, ist die Wirkkraft der Metapher vom umfassenden „Meer" an Informationen, das die digitale Surferin trägt, in dem sie aber ebenso rasch ertrinken kann. Die Informations- „Tropfen“ fügen sich nahtlos zum Daten-Ozean zusammen, der über dem überforderten Individuum zusammenschlägt. Bruchstückhafte Informationen scheinen hier das Problem nicht zu sein - im Gegenteil. 
Das letzte Mal begegnete mir der Begriff des Fragments in Gestalt jener Defragmentierung, welche ich meiner auf der Festplatte abgelegten Datenstruktur zugute kommen lassen sollte. Doch seit die magnetischen Datenträger durch SSD (Solid State Drives) alias "Flash-Speicher“" abgelöst worden sind, ist das Fragment semantisch auch aus diesem Teil der digitalen Welt verschwunden (de.wikipedia.org/wiki/Fragmentierung_(Dateisystem)).

Das Fragment mutet im Reden vom digitalen Netz als Fremdkörper an. In der Netzmetapher können Fragmente nur Ausdruck des Scheiterns, des Versagens darstellen: Ein fragmentiertes Netz ist ein dysfunktionales oder gar zerstörtes Netz. So gesehen kann das Fragment als Antithese, als Irritation oder Störung des idealisierenden Netz-Konzepts verstanden werden. Bekanntlich gehören Störungen und Irritationen zu jedem Ideal, so auch im Netz, das blitzschnell fragmentiert werden kann, wenn die digitalen Verbindungen zwischen Maschinen, Menschen, Daten abbrechen.

Auch wenn der Begriff des Fragments im digitalen Umfeld fremd, ja anachronistisch wirken mag: Fragmente - das die Behauptung dieses Beitrags - sind im hier zu behandelnden Bereich, der historischen Blogosphäre, allgegenwärtig. Ja, mehr noch: Fragmente stellen in verschiedener Hinsicht ein konstitutives Element der gebloggten Historiographie dar.

Ist das Fragment wirklich eine „Störung“ im Konzept der Vernetzung? Das Internet zumindest baut funktional auf Fragmentierung auf. Das TCP/ IP-Protokoll tut nichts anderes, als Informationen in kleine Dateneinheiten zu zerteilen und die so entstandenen Fragmente als Datenpakete durch das Netz zu schicken, wo sie empfängerseitig wieder zu einem Ganzen zusammengebaut werden. Misslingt dieser Vorgang, erhält der Empfänger ein Fragment (www. rvs.uni-bielefeld.de/ heiko/tcpip/tcpip_html_alt/kap_2_3.html). Allerdings handelt es sich hier um ein Fragment einer Kopie, das Resultat einer unvollständig ausgeführten Duplikation. Daher lässt sich diese Störung einfach beheben durch eine neuerliche Anforderung des fehlenden Datenpakets. Nur am Rande sei erwähnt, dass zuweilen die IP-Pakete unterwegs noch einmal geteilt, also fragmentiert werden können, wenn das Paket bei der Übermittlung auf ein Nadelöhr stößt, durch das es nicht hindurch passt (stack.nil.si/ipcorner/IP_ Fragmentation/). 
Fragmente sind Teile eines nicht vorhandenen Ganzen, auf das sie in ihrer Unvollständigkeit verweisen. Dieser Verweis kann dabei auf das nicht mehr oder noch nicht Vorhandensein der Totalität Bezug nehmen (Schmidt 2003, 66). Fragmente haben folglich zwei Bedeutungsrichtungen: Sie stellen zum einen einen unvollständigen Überrest eines ehemals Ganzen dar, das zerstört oder verloren gegangen ist. Im Vordergrund steht hier die Wahrnehmung des Verlusts, des „nicht mehr“. Doch Fragmente können zum anderen auch als Teile eines noch unfertigen Ganzen gesehen werden. Hierbei dominiert die Wahrnehmung des Fragments als Potentialität, des „noch nicht“. In jedem Fall verweisen Fragmente auf ein Ganzes, das nur skizziert und angedeutet wird und das im Umgang mit den Fragmenten imaginiert werden muss. Hier eröffnen sich Spielräume für eine Vorstellungskraft, die Möglichkeiten abzuwägen und nicht nur Gewissheiten zu konstatieren in der Lage ist.

Die Dichotomie von Fragment und Totalität wirft grundlegende erkenntnistheoretische und philosophische Fragen auf: Wie hängen Fragment und Totalität zusammen, sind sie ohne einander denkbar, sind sie je alleine denkbar? Die Beziehung zwischen Teil und Ganzem sind für Dällenbach und Hart Nibbrig ausschlaggebend für das Wesen des Fragments. Sie unterscheiden drei Typen. Zum einen führen sie die bereits genannte Vorstellung eines Fragments als „Teil eines Ganzen, dem es in zeitlicher Hinsicht nicht mehr oder noch nicht angehört und für dessen Abwesenheit es in stückhafter Präsenz einsteht [...] als pars pro toto“ an. Je nachdem kann es folglich „als Rest, Abfall [...] Spur, Ruine, Memorandum oder [...] als Sprungbrett für die Phantasie" dienen (Typus 2). Daneben sehen sie aber auch jene Fragmente „als Teil eines Ganzen, dessen Vollständigkeit nicht in Frage steht“, wodurch ein wesentlicher „Charakter des Fragmentum, das [...] Abgerissensein vom Ganzen" fehlt und die Eigenschaft des Fragments grundsätzlich in Frage steht (Typus 1). Und schließlich nennen Dällenbach und Hart Nibbrig eine dritte Auffassung, bei der eine Totalität weder vorstellbar noch (wieder-)herstellbar ist (Typus 3; alle Zitate Dällenbach/ Hart Nibbrig 1984, 15). Beim letzten Typus löst sich die Dichotomie von Fragment und Totalität auf und wirft die Frage auf, ob das Fragment überhaupt noch als Fragment bezeichnet werden kann.

Gegen Dichotomien wandten sich Deleuze und Guattari mit ihrem Konzept der Rhizome. Verbindungen von einzelnen Teilen entwickeln sich in dieser Theorie dynamisch und sind folglich nicht als festgefügte Struktur zu verstehen. Vor allem entwickeln sich Rhizome frei von einer übergeordneten, als Gesamtheit zu verstehenden Idee, welche die Wuchs- und Entwicklungsrichtungen 
vorgeben (Deleuze/Guattari 1977). Im Rhizom, so scheint es, treffen sich die so gegensätzlichen Konzepte von Fragment und Vernetzung. Die HypertextTheorie (z. B. Landow 2006, 60) hat sich das Rhizom als Gegenkonzept zur hierarchischen Baumstruktur gleichsam einverleibt, was nicht ohne kritisches Echo geblieben ist (Buchanan 2008). Bedeutsam für die hier vorliegenden Überlegungen ist die Feststellung einer fragmentierenden Logik der hypertextuellen Vernetzung: Um einen Text als Netz oder Gewebe zu schaffen, das in seiner Struktur den Rezipient/innen nicht vorgibt, wie (d.h. insbesondere in welcher Reihenfolge und damit mit welcher Sinnstruktur) es rezipiert werden soll, muss besagter Text erst zerteilt werden (Bolz 1993, 208). Fragmentierung ist Voraussetzung für Vernetzung.

Die Ambivalenz des Fragments, die sich in seinen zwei Verweisrichtungen "nicht mehr“ und „noch nicht“, aber auch in der letztlich „unfassbaren“ Dialektik von Teil und Ganzem manifestiert, lässt sich auch verwenden, um den Erkenntnisprozess in den Geschichtswissenschaften zu beschreiben. Geschichte befasst sich mit Fragmenten, die aus der Vergangenheit übriggeblieben sind. Die Geschichtswissenschaften nennen sie „Quellen“. Wiewohl diese Quellen Spuren und Überreste darstellen, mithin also auf das "nicht mehr" verweisen, so verweist das Wort „Quelle“ zugleich auf den „noch nicht"-Charakter der Fragmente und betont damit ihre Bedeutung als Grundlage der Geschichtsschreibung.

Linguistic und Narrative Turn (vgl. Noiriel 2002) haben vor Augen geführt, dass Geschichte ein Konstrukt darstellt, das die gegenwärtige vielfältige Gliederung der Gesellschaft und der damit verbundenen Interessen abbildet. Geschichte stellt Vergangenheit nicht wieder her und kann sie auch nicht abbilden, wie sie wirklich war. Geschichtsschreibung muss sich - unter Berücksichtigung wissenschaftlicher Plausibilitäts- und Wirklichkeitsansprüche - jenen imaginativen und interpretatorischen Spielraum im Umgang mit Fragmenten schaffen, der bedeutungsvolle historische Sinnbildungen überhaupt erst ermöglicht. Aus Fragmenten werden Geschichten.

Im Akt der Geschichtsschreibung werden wohl Fragmente durch Interpretations- und Kontextualisierungsleistungen zu einer sinnvollen Geschichte zusammengefügt. Solche Geschichtsdarstellungen bilden, wenn sie gelungen sind, ein überzeugendes Ganzes. Und doch bleibt auch Geschichte als Unterfangen immer den Prämissen der Selektivität und Partikularität unterworfen. Aus diesem Grund „muss, wer etwas erzählt, auswählen, weglassen und das Erzählte 
in einer Geschichte anordnen, die chronologisch oder sachlich angeordnet ist" (Baberowski 2005, 213). Jede Geschichte muss sich auf ein Set von Akteuren, Ereignissen, Vorgängen, Zuständen beschränken und diese aus einer bestimmten Perspektive in einer Erzählung zusammenfügen. Insofern bleibt jede Erkenntnis über die Vergangenheit und damit jede Geschichtsschreibung selbst ein Fragment, das auf das Ganze der nie vollumfänglich zu erfassenden Vergangenheit verweist.

$\mathrm{Zu}$ Beginn des 21. Jahrhunderts ist eine Zergliederung der Geschichte als Ausdruck einer historiographischen Entwicklung festzustellen, die in den letzten Jahrzehnten zu einer Abwendung von nationalen oder eurozentrischen Meistererzählungen und $\mathrm{zu}$ einer Hinwendung zu Untersuchungen unter spezifischen Fragestellungen gesellschaftlicher Teilgruppen oder zu einer umfassenden Globalgeschichte geführt hat. Die Geschichte sieht sich dabei konfrontiert mit „der widersprüchlichen Forderung nach Einheit auf der einen und Fragmentierung auf der anderen Seite“ (Völkel 2006, 346).

Fragmente sind jedoch nicht nur eine erkenntnistheoretische Denkfigur, sondern auch eine literarische Textform. Ende des 18. Jahrhunderts als literarische Form etabliert verfassen bedeutende Schriftsteller besonders des frühen 20. Jahrhunderts wie Musil, Döblin oder Kafka ihre Werke in fragmentarischer Form. Dies lenkt das Interesse auf die Geschichtsschreibung als auktorialen Text. Liegen auch Geschichtsdarstellungen in fragmentarischer Form vor?

Walter Benjamin beabsichtigte seine historische Darstellung über das Paris des 19. Jahrhunderts („Passages“) in einer fragmentarischen Form zu veröffentlichen. Dies war aber zugleich auch ein Ausdruck seiner Ablehnung herkömmlicher Geschichtswissenschaft. Er wandte sich dezidiert gegen die „sinngestützt-hermeneutische und auf einen Kontinuitätszusammenhang verpflichtete traditionelle Geschichtsdarstellung" (Greiert 2011, 513). Darin, so war Benjamin überzeugt, würde nur die Geschichte der Sieger repliziert. Die Geschichte der Besiegten müsste in einem Modus erzählt werden, der keine Sinndeutungen vorgebe. Wie genau eine solche Geschichtsschreibung nach Meinung von Benjamin letztlich aussehen sollte, bleibt unklar, denn Benjamin nahm sich vor der Vollendung der "Passages“ auf der Flucht vor den nationalsozialistischen Truppen 1940 an der französisch-spanischen Grenze das Leben (www.gdw-berlin.de/nc/de/vertiefung/biographien/biografie/viewbio/benjamin/). Der letzte Text, an dem Benjamin vor seinem Tod arbeitete, 
war sein fragmentarischer Text „Über den Begriff der Geschichte“ (www. mxks.de/files/phil/Benjamin.GeschichtsThesen.html), der den „Passages“ vorangestellt werden sollte. Bekannt wurde aus diesem Text jenes Fragment, in dem er ausgehend von einem Bild Paul Klees den „Engel der Geschichte“ beschreibt.

Während des Zweiten Weltkrieges entstand ein weiteres geschichtsphilosophisches Fragment, Marc Blochs „Apologie der Geschichtswissenschaft oder der Beruf des Historikers" (Bloch 1949/2002). Bloch kämpfte im Untergrund und wurde von der Gestapo umgebracht, bevor er sein Werk vollenden konnte. Bloch schrieb den Text im unbesetzten Teil Frankreichs und war von seiner Bibliothek in Paris getrennt. Man könnte in fragwürdiger Analogie zum Schluss kommen, sein Wissensnetz sei fragmentiert und der Zugriff darauf unterbrochen gewesen.

Die erkenntnistheoretischen und historiographiegeschichtlichen Überlegungen zu Fragmenten und ihre Bedeutung für die Geschichte sind hier von Belang, weil der digitale Medienwandel mit dem Anspruch einhergeht, völlig neue Kommunikations- und Diskursformen zu ermöglichen, die den „Abschied von Gutenbergs Welt der Schrift" bedeuten (Bolz 1993, 183). Im hier interessierenden Falle geht es um die Frage, ob Weblogs völlig neue, noch nie da gewesene Formen der Geschichtsschreibung ermöglichen, und falls diese Frage bejaht wird, welche Konsequenzen dieser Befund für den geschichtswissenschaftlichen und (ausserhalb des fachinternen Austausches) für den geschichtskulturellen Diskurs hätte.

Was Benjamin ablehnte, ist spätestens seit Hayden Whites Betrachtungen zur metahistory (White 1986) im geschichtstheoretischen Diskurs etabliert: Die Erzählung in ihrer typischen Ausprägung mit Anfang, Peripetie und Ende ist die Standardform der Geschichtsschreibung. In Erzählungen ist das Ende eines Geschehens bereits bekannt, nur so lassen sich die Ereignisse, Entscheidungen, Prozesse und Zustände in einen erklärenden, sinnvollen Deutungszusammenhang stellen. In Erzählungen wird aus der Kontingenz vergangener Ereignisse, Vorgänge und Zustände eine sinnvolle Geschichte. Inwiefern können Geschichtsdarstellungen fragmentiert sein, ohne sinnlos zu werden? 
Gumbrechts Werk „1926“ (Gumbrecht 2001) gibt uns hier interessante Aufschlüsse. Gumbrecht fügt eine Reihe kurzer Darstellungen zu Geschehnissen, Vorstellungen, Personen und sozioökonomischen Strukturen zusammen, die er mit dem Jahr 1926 in Verbindung gebracht hat. Auf den ersten Blick wirkt dies wie eine zufällige zusammen gewürfelte Anhäufung von historiographischen Fragmenten. Es ist das Ergebnis einer sorgfältigen Auswahl von Inhalten, die einer übergeordneten Idee folgt. Diese Fragmente sind (im Sinne der Typologie von Dällenbach/Hart Nibbrig) eher dem Typus 1 zugehörig. Diese Einschätzung bestärken die von Gumbrecht bei den einzelnen Fragmenten angebrachten Verweise, die zu anderen Fragmenten führen. Auch sie sind Ausdruck einer Auswahl, einer Entscheidung, die auf eine übergeordnete Idee verweisen, wie Geschichte präsentiert werden soll. Diese Idee liegt dem Entwurf des Buches zugrunde, und sie findet ihren Abschluss beim Druck des Buches. Gumbrechts „1926“ ermöglicht trotz seines fragmentarischen Charakters keine vollends offene Rezeption. Denn auch wenn die Reihenfolge, in welcher sie es tun, den Lesenden freisteht: rezipieren können sie nur, was Gumbrecht im Buch vorgelegt hat.

Die Technologie des Hypertexts wirft die Frage auf: Gibt es nicht-lineare Geschichtsdarstellungen? Denn ein wesentliches Merkmal von Hypertexten ist die Möglichkeit, unsequenzierte Texte zu erstellen, zu deren Rezeption nicht eine vom Autor festgelegte Reihenfolge eingehalten werden muss. Wie Krameritsch (2007) zeigt, führt dies zu einer Verschiebung der Gestaltungsmöglichkeiten des konkret zu rezipierenden Textes von den Autor/innen zu den Lesenden. Diese erhalten somit mehr Gestaltungsmöglichkeiten, aber auch mehr Verantwortung in Bezug auf die Geschichte, die sie in Hypertexten rezipierend herstellen.

Dennoch betont Krameritsch die Verantwortung der Autor/innen für die Kohärenz von Hypertexten. Die innere Stimmigkeit wird durch die Verständlichkeit der einzelnen Module bestimmt, die gewährleistet sein muss - unabhängig davon, welche anderen Module die Lesenden zuvor gelesen haben. Sie wird aber vor allem hergestellt durch sinnvolle Verbindungen zwischen den Modulen, durch Hyperlinks. Die Links drücken die Auffassung der Autor/innen aus, wie die gesamte Geschichtserzählung kohärent, und damit sinnvoll zu einem Ganzen gefügt werden kann. Die Freiheit der Lesenden besteht in der Wahl der Verbindungen, nicht in deren Herstellung. Und diese hypertextuellen Geschichtsdarstellungen haben mit Fragmenten folglich wenig gemein. 
Links in hypertextuellen Geschichtsdarstellungen wirken sinnstiftend und sind Ausdruck einer erzählerischen Absicht, auch wenn sich diese nicht in einer linearen Darstellungsform manifestiert. Dies zeigt gerade der Bruch solcher Konvention in den grotesk wirkenden Zusammenstellungen von WikipediaArtikeln in der Rubrik „in sieben Schritten“ im Online-Portal „einestages“ (einestages.spiegel.de/rubrik/In-sieben-Schritten). Die humorigen Beiträge unter Titeln wie „Wie der Inzest römischer Kaiserfamilien zum Triumph der Piratenpartei führte“ oder „Wie Mark Twain das Burnout erfand“ führen die populäre Wahrnehmung, dass in Geschichte irgendwie Alles mit Allem zusammenhängt, ad absurdum. Sie machen sich hierfür die Eigenschaften der kollaborativ erstellten Online-Enzyklopädie Wikipedia zu Nutze, wo die Benutzer/innen nicht nur selber die Einträge verfassen, korrigieren und erweitern, sondern auch nach eigenem Dafürhalten Links zu anderen Einträgen anlegen können.

Wikipedia ist ein bestechendes Beispiel für einen „offenen Hypertext“, wie Krameritsch ihn bezeichnet hat (Krameritsch 2007, 151-157). An offenen Hypertexten können die Lesenden aktiv mitwirken, er wird laufend weiterentwickelt, denn er stellt nicht ein abgeschlossenes, fertiges Produkt dar. Wikipedia ist auch im Hinblick auf die Darstellung von Geschichte ein "offener Hypertext“: es gibt keine übergeordnete Idee einer Erzählung, nach der sinnbildend eine Auswahl getroffen werden kann, die die Einzelteile zu einem bedeutungsvollen Ganzen verbindet.

Offene Hypertexte können, solange sie offen sind, keine Erzählungen sein, denn ihnen fehlt das Ende. Sie können - wie das Beispiel der Rubrik „In sieben Schritten“ (einestages.spiegel.de/rubrik/In-sieben-Schritten) zeigt - von den Lesenden durch Auswahl und Anordnung von Teilen mit einem Ende versehen und dadurch zu einer Geschichte geformt werden. Ohne diesen Eingriff der Lesenden bleiben die Teile der Wikipedia letztlich, so intensiv sie verlinkt und Teil der "ganzen Enzyklopädie“ sein mögen, im Hinblick auf geschichtliche Sinnbildung nichts anderes als Fragmente.

In dieser Hinsicht ist Wikipedia ein besser organisiertes, einheitlicher formatiertes Abbild des World Wide Web selbst, das auch einen großen, offenen Hypertext darstellt, aus dem die Lesenden durch Selektion und Anordnung von einzelnen Fragmenten sich abhängig von den konkreten Erkenntnisbedürfnissen eine "Geschichte“ erstellen müssen. Diese Konstellation birgt 
die Möglichkeit der völligen Fragmentierung der Geschichte in individuelle Geschichten, die jegliche intersubjektive Verständigung verunmöglicht. Krameritsch führt daher den Modus des „situativen Erzählens" ein, der zumindest zeitweise eine kollektive Verständigung über Vergangenheit ermöglichen soll, um Orientierung für zukünftiges Handeln zu schaffen (Krameritsch 2009). Schmale skizziert den gegenwärtigen medialen Kontext der Geschichtswissenschaften als von „Hybridität, Volatilität, Fluidität, Hypertextualität“ geprägt, was zur Herausforderung - auch an die Geschichtwissenschaften - führe, „in einer wie noch nie in Fluss geratenen Welt Kohärenz zu erzielen" (Schmale 2010, 116).

Geschichts-Weblogs sind Beiträge von unterschiedlichen Individuen zum "offenen Hypertext" des geschichtswissenschaftlichen und/oder geschichtskulturellen Diskurses. Dass die Grenze zwischen Amateur/in und professional, zwischen Einzelmaske und Vertreter einer Institution, zwischen akademischallgemeingültigen Wissenschaftsanspruch und subjektiver Befindlichkeit hier unklar verläuft, ist hinlänglich bekannt und je nach Sichtweise und Interesse beklagt oder begrüßt worden. Doch selbst wenn die Geschichtswissenschaftler/ innen ihren Anspruch auf ungestörte professionelle Auseinandersetzung mit Vergangenheit in virtuellen gated communities durchsetzen können, wird der zunehmende Gebrauch von Weblogs zu einer Fragmentierung des Diskurses in unzählige Einzelstimmen führen. Dies wird neue Techniken der diskursiven Orientierung (gleichsam der „Defragmentierung“) erfordern, die wiederum stark an konkreten Bedürfnissen von Einzelnen oder von Gruppen ausgerichtet sein dürften. $\mathrm{Zu}$ beachten bliebe auch, dass sich diese Fragmentierung keineswegs egalitär vollzieht. Möglicherweise bilden sich die alten Hierarchien des etablierten, vor-digitalen Feldes nicht unmittelbar im hypertextuellen Diskurs ab, doch auch dieser Diskurs wird von Hierarchien geprägt: nicht jedes Blog erhält die gleiche Aufmerksamkeit und hat den gleichen Einfluss (Puschmann 2012).

Eignen sich Weblogs für Geschichtsdarstellungen, oder, mit anderen Worten: Kann man in Weblogs Geschichte erzählen?

Weblogs sind Hypertexte. Es entbehrt nicht einer gewissen Ironie, dass Nicht-Linearität als ein Kernmerkmal von Hypertexten gilt (Kuhlen 1991), Weblogs jedoch (zumindest in der Standardeinstellung) die einzelne Beiträge linear, nämlich in umgekehrt chronologischer Reihung darbieten - und dies zwingend durch die Programmierung der Blogsoftware (Puschmann 2012). 
Durch diese technische Vorgabe liegen Weblogs in sequenzierter Form vor; sie bieten den Lesenden eine klare Leserichtung. Diese ist allerdings narrativ „verkehrt", nämlich vom Ende zum Anfang hin angeordnet. Sie eignen sich folglich - wie gleich ausgeführt werden soll - in ihrer Grundanordnung nicht für Erzählungen im herkömmlichen Sinn, und folglich auch nicht für konventionelle Geschichtsdarstellungen.

Weblogs sind offene Hypertexte. Sie sind dies weniger, weil sich die Lesenden aktiv beteiligen könnten. Das wäre zwar möglich, wird aber abgesehen von Kommentarfunktionen kaum realisiert. Weblogs sind offene Hypertexte, weil ihr Ende buchstäblich „offen“ ist. Zwar gibt es Projekte, die den Tagebuch-Charakter von Weblogs nutzen, um historische Ereignisse in „Echtzeit" abzubilden, bzw. nachzubilden. Als gelungenes Beispiel kann das Projekt Pepys Diary (www.pepysdiary.com) betrachtet werden. Hierbei ist das Ende des Weblogs zu Beginn bereits bekannt (und das Weblog wird dann eingestellt). Doch wesentliche Eigenschaft eines Weblogs ist die Unbestimmtheit seines Endes. Die Erzähltheorie hingegen ist der Ansicht: „Wer erzählt, muss einen Anfang und ein Ende finden, in deren Mitte sich etwas Erzählenswertes ereignet. [...] Dabei ist zu berücksichtigen, dass erst das Ende den kompositorischen Anfangspunkt einer Handlung gewissermaßen ex post bestätigt, dass die narrative Struktur also nicht einfach linear und progredierend, sondern in hohem Maß rekusiv angelegt ist" (Koschorke 2012, 61). Hier eröffnet sich folglich eine Diskrepanz zwischen erzähltheoretischen Anforderungen an historische Darstellungen und medienspezifischen Voraussetzungen von Weblogs, es sei denn - wie im Falle von Pepys Diary - das Ende ist bei Beginn des Weblog-Projekts bereits bekannt. Weblogs als offene Form fortlaufenden Publizierens eignen sich folglich nicht für Geschichtsdarstellungen im herkömmlichen Sinn. Sie ermöglichen allenfalls die Publikation einer Serie von singulären Darstellungen, die (im Stile von Gumbrechts „1926“) beliebig kombiniert und durch Links verbunden werden können.

Da Weblogs den etablierten narrativen Modus von Geschichtsschreibung nicht einfach außer Kraft setzen können, wird in Weblogs keine Geschichte geschrieben - zumindest nicht im herkömmlichen Sinne. Dennoch gibt es unzählige Weblogs, die Geschichte zum Thema haben. Eine umfassende Analyse aller Geschichtsblogs steht noch aus, dennoch sei die Aussage gewagt, dass diese Blogs sich in den meisten Fällen mit Geschichte auf einer Meta-Ebene befassen. Das heißt, dass die Autor/innen von Geschichtsblogs entweder grundsätzliche Fragen der Geschichtswissenschaft (z. B. edwired, edwired.org) oder der Ge- 
schichtsdidaktik (z. B. Medien im Geschichtsunterricht, geschichtsunterricht. wordpress.com) im Zusammenhang mit digitalen Medien diskutieren, über Erkenntnisse und Überlegungen im Rahmen eigener Forschungsinteressen oder Forschungsvorhaben (z. B. Adresscomptoir, adresscomptoir.twoday. net) berichten und allenfalls Ausschnitte aus diesem Arbeitszusammenhang publizieren (z. B. Georgian London, www.georgianlondon.com), oder Blog-Einträge, die im klassischen Sinne Geschichte erzählen, zumindest kommentieren (z.B. renaissance mathematicus, thonyc.wordpress.com, oder Geschichtsblog, geschichts-blog.blogspot.ch). Denn einzelne Blogposts, unter Umständen sogar in kleinen Serien, eignen sich durchaus für die Darstellung von Geschichte. Hierin kann die Flexibilität des technischen Formats, das verschiedene Genres ermöglicht (Puschmann 2012) voll ausgenützt werden. Doch Weblogs als „Gesamtformat" eignen sich, es sei ein weiteres Mal betont, nicht für Geschichtsdarstellungen im herkömmlichen Sinn.

Weblogs, die Geschichte thematisieren, bestehen folglich aus Blogposts, die in unterschiedlicher Weise Ergebnisse historischer Erkenntnis darstellen oder den Prozess und die Rahmenbedingungen der Erkenntnis diskutieren. Diese Posts sind als Fragmente zugleich isoliert als auch verbunden. Sie können von den Lesenden auch in unterschiedlichen Formen angeordnet werden: in ausgewählten chronologischen Ausschnitten („Archiv“), sortiert nach Kategorien oder Tags (Stichworten) des Autors bzw. der Autorin oder nach einem eigenen Suchbegriff. Die Blogposts sind in verschiedene Kontexte eingebunden: mittels Anordnung durch die Blog-Chronologie in die Selbstdarstellungsbemühungen des Bloggers, bzw. der Bloggerin; durch Verweise (seien es Hyperlinks oder Literaturnachweise) in den geschichtswissenschaftlichen Diskurs zum jeweils behandelten historischen Narrativ; mittels Indexierung in die algorithmische Suchlogik von Suchmaschinen; mittels Empfehlung auf Social-Media-Plattformen („I like“, „Tweet this“) in soziale Netzwerke der Leserschaft. Die Bezugsgröße des Fragments bleibt somit unbestimmt, variabel und situationsbezogen. Im „pluralistischen Kontext [der Postmoderne] verliert die Form des Fragments ihre Verweisungskraft" (Ostermann 2003, 47). Das Fragment bleibt isoliert, geht gleichsam „verloren“ - und mit ihm seine historisch-narrative Funktion und historiographische Bedeutung.

In derlei narrativen Fragmenten digitaler Geschichtsdarstellungen werden den Usern keine klaren narrativen Zusammenhänge mehr offeriert; die Herstellung von Deutungszusammenhängen wird vielmehr den Usern überlassen. Dies kann durchaus eine Chance für die Etablierung neuer, weniger starrer 
und idealerweise reflektierter Geschichtsbilder darstellen. Es wäre lediglich zu prüfen, ob durch solche narrativen Fragmente, wie sie sich etwa in Weblogs manifestieren, nicht einfach allgemein gängige Deutungsmuster konventioneller Narrative latent transportiert und befestigt werden. Die Bezugsgrößen verschwinden, die Deutungen bleiben. Dabei wäre doch das Herstellen von historiographischer Kohärenz, so Schmale (2010), eine wichtige Aufgabe der Geschichtswissenschaft - auch wenn keine dauerhafte, sondern nur eine temporärer oder (nach Krameritsch 2009) „situative“ Kohärenz sein kann.

In Weblogs lässt sich keine Geschichte erzählen. Die Hoffnung auf neue Formen einer vernetzten, offen strukturierten, modularisierten Geschichtsschreibung bildet sich bislang in den bekannten historisch interessierten Weblogs nicht ab. Eine solche Geschichtsschreibung aus narrativen Fragmenten, die auch schon erprobt wurde, aber sich nicht etabliert hat (vgl. das 2004 mit dem Medidaprix ausgezeichnete, nicht mehr online erreichbare Projekt pastperfect.at), wird die Weblogs mit den Tücken der Kohärenzbildung und dem erzähltheoretischen Problem des "offenen Endes" konfrontieren. Währenddessen zeigt die Praxis der Weblog-Verwendung jedoch eine andere, ebenso wichtige Funktion im geschichtswissenschaftlichen Diskurs auf. In ihnen wird laufend und vielseitig berichtet von Menschen in ihrem Bemühen, Geschichte zu erzählen. Mit anderen Worten: Die Bedeutung von Weblogs für die Geschichte besteht weniger in neuen Formen der Darstellung von Geschichte, sondern in der kommunikativen Verhandlung der Personen, die Geschichte erforschen und schreiben, und der Rahmenbedingungen, unter denen sie dies tun.

\section{Literatur}

Marc Bloch: Apologie der Geschichtswissenschaft oder der Beruf des Historikers [1949]. Aus dem Französischen von Wolfram Beyer. Mit Annotationen und einem Nachwort von Peter Schöttler, Stuttgart: Klett 2002.

Norbert W. Bolz: Am Ende der Gutenberg-Galaxis. Die neuen Kommunikationsverhältnisse. München 1993.

Ian Buchanan: Deleuze and the Internet, in: deleuze international 1 (2008), [deleuze.tausendplateaus.de/wp-content/uploads/2008/01/deleuze-and-theinternet.pdf], eingesehen 5.10.2012. 
Lucien Dällenbach/Christiaan Lucas Hart Nibbrig: „Fragmentarisches Vorwort“, in: Dies. (Hrsg.): Fragment und Totalität (Edition Suhrkamp, 1107). Frankfurt a.M 1984, S. 7-17.

Gilles Deleuze: Rhizom (Internationale marxistische Diskussion Bd. 67). Berlin 1977.

Andreas Greiert: Erlösung der Geschichte vom Darstellenden. Grundlagen des Geschichtsdenkens bei Walter Benjamin 1915-1925. München 2011.

Hans Ulrich Gumbrecht: 1926. Ein Jahr am Rand der Zeit. Frankfurt a. M. 2001. Albrecht Koschorke: Wahrheit und Erfindung. Grundzüge einer allgemeinen Erzähltheorie. Frankfurt a.M 2012.

Jakob Krameritsch: Die fünf Typen des historischen Erzählens im Zeitalter digitaler Medien, in: Zeithistorische Forschungen/Studies in Contemporary History 6/3 (2009), S. 413-432.

Jakob Krameritsch: Geschichte(n) im Netzwerk. Hypertext und dessen Potenziale für die Produktion, Repräsentation und Rezeption der historischen Erzählung. Münster 2007.

George P Landow: Hypertext 3.0. Critical theory and new media in an era of globalization. Baltimore, Md 2006.

Jean-François Lyotard/Peter Engelmann: Das postmoderne Wissen. Ein Bericht (franz. Original: La condition postmoderne, Paris 1979). Wien 2009.

Gérard Noiriel: Die Wiederkehr der Narrativität, in: Joachim Eibach/Günther Lottes (Hrsg.): Kompass der Geschichtswissenschaft. Göttingen 2002, S. $355-$ 370 .

Eberhard Ostermann: Das Fragment in der Postmoderne, in: Bernhard Fetz/ Klaus Kastberger (Hrsg.): Die Teile und das Ganze Bausteine der literarischen Moderne in Österreich (Profile (Zsolnay), Bd. 10). Wien 2003, S. 44-52.

Cornelius Puschmann: Technisierte Erzählungen? Blogs und die Rolle der Zeitlichkeit im Web 2.0, in: Nünning, A u. a. (Hrsg.): Narrative Genres im Internet: Theoretische Bezugsrahmen, Mediengattungstypologie und Funktionen. Trier 2012, S. 93-114.

Wolfgang Schmale: Digitale Geschichtswissenschaft. Wien 2010.

Burghart Schmidt: Fragment/Ruine-Die Zeichenweise dieser Zeit. Auch Weltstand?, in: Bernhard Fetz/Klaus Kastberger (Hrsg.): Die Teile und das Ganze Bausteine der literarischen Moderne in Österreich (Profile (Zsolnay), Bd. 10). Wien 2003, S. 63-80. 
74 Jan Hodel

Markus Völkel: Geschichtsschreibung. Eine Einführung in globaler Perspektive. Köln 2006.

Hayden White: Auch Klio dichtet oder die Fiktion des Faktischen. Studien zur Tropologie des historischen Diskurses. Stuttgart 1986. 